\title{
Improved Detector for Continuous Wave Radar Waveforms exploiting Phase Information
}

\author{
Johannes Fink*, Stefan Wunsch*, Friedrich K. Jondral \\ Communications Engineering Lab, Karlsruhe Institute of Technology \\ Johannes.Fink@kit.edu, Stefan.Wunsch@student.kit.edu, Friedrich.Jondral@kit.edu
}

\begin{abstract}
By transmitting multiple copies of an arbitrary (frequency modulated) continuous wave radar signal, phase information in target echoes can be exploited for detection. The phase information is gained by calculating the sample variance of phase spectra of the multiple echoes. In this manner, phase and amplitude information is available at the detector. The optimal detector decides based on two-dimensional decision regions in the amplitude/phase-variance space. Suboptimal detectors combine two one-dimensional decisions. It is shown, that the proposed method improves detection performance especially at low signal to noise ratios. The optimal detector achieves a gain in detection rate compared to the conventional square law detector of nearly $10 \%$ at a signal to noise ratio (SNR) of $3 \mathrm{~dB}$. The performance gain reduces to about $3 \%$ at a SNR of $9 \mathrm{~dB}$. One of the suboptimal detectors also outperforms the conventional detector, but achieves lower gains in detection rates than the optimal detector.
\end{abstract}

Keywords-Detection, CW, FMCW, Radar, DSP, ROC, Waveform Design

\section{INTRODUCTION}

A figure of merit representing the performance of a radar detector is the Receiver Operating Characteristic (ROC). The ROC shows the interrelation between detection rate (DR) and false alarm rate (FAR) at a given signal to noise ratio (SNR) for the given radar detector.

It is known, that in theory, the optimal radar detector is the coherent one, which makes use of both amplitude and phase information of the received signal [1]. However, usually in radar and especially when using (frequency modulated) continuous wave radar signals, it is difficult to make use of the phase information. Thus, practical detectors like the linear or the square law detector base their decisions solely on the information contained in the amplitude of the signal [1].

In this paper, a new method for detection is proposed, which alters the radar waveform in such a way that the phase information can be exploited for detection. This is achieved by multiple transmission of the same waveform and jointly processing the multiple echoes. The multiple transmissions obey an energy constraint, such that a fair comparison with conventional detectors is possible.

\section{Signal Modell}

We assume a continuous wave radar signal which may or may not be frequency modulated. Let $f_{\mathrm{WF}, i}(t)$ be the

\footnotetext{
${ }^{*}$ These authors contributed equally to this work.
}

instantaneous frequency of signal $i$ at time $t$, then

$$
s_{i}(t)=a_{\mathrm{s}, i} \cos \left(2 \pi \int_{0}^{t} f_{\mathrm{WF}, i}(\tilde{t}) \mathrm{d} \tilde{t}+\phi_{0, i}\right)
$$

is the real signal being transmitted, where $\phi_{0, i}$ is the starting phase at $t=0$ and $a_{\mathrm{s}, i}$ is the amplitude of the signal. The variable $i$ is used for enumeration and will be needed in order to introduce the modified signal. Waveforms which can be modeled by (1) are very popular for low-cost radars, as they can be implemented using a voltage controlled oscillator (VCO) and a homodyne receiver architecture and currently experience much attention in industrial and automotive applications [2]. In the following we will focus on linear frequency modulated (LFM) waveforms [3], where

$$
f_{\mathrm{WF}, i}(t)= \begin{cases}\frac{B}{T} t+f_{i}=\alpha t+f_{i}, & 0 \leq t<T \\ 0, & \text { otherwise }\end{cases}
$$

with $B$ beeing the sweep bandwidth, $T$ the chirp duration and $f_{i}$ the lower end of the used frequency band. For the case of no frequency modulation, which is commonly known as single frequency continuous wave $(\mathrm{CW})$ radar or doppler radar, in (2), let $B=0 \Rightarrow \alpha=0 \Rightarrow f_{\mathrm{WF}, i}(t) \equiv f_{i}$.

The energy $E_{T, i}$ of waveform $i$ can be expressed in terms of the energy $E_{T, i, 1}$ of the waveform with unit amplitude:

$$
E_{T, i}=\int_{0}^{T}\left|s_{i}(t)\right|^{2} \mathrm{~d} t=a_{\mathrm{s}, i}^{2} E_{T, i, 1}
$$

Assuming a point target at a distance $R$, which is moving at constant radial velocity $v_{\mathrm{r}}$ towards the radar, the echo signal

$$
e_{i}(t)=a_{\mathrm{e}, i} \cos \left(2 \pi \int_{0}^{t}\left(f_{\mathrm{WF}, i}(\tilde{t}-\tau)-f_{\mathrm{D}, i}\right) \mathrm{d} \tilde{t}+\phi_{0, i}\right)
$$

is a time and frequency shifted version of (1) with

$$
\tau=\frac{2 R}{c}
$$

being the two-way time delay and

$$
f_{\mathrm{D}, i} \approx \frac{2 v_{\mathrm{r}}}{c}\left(f_{i}+\frac{B}{2}\right)
$$

the doppler shift. The approximation in (6) holds for $v_{\mathrm{r}} \ll c$ and $B \ll f_{i}$. Furthermore $a_{\mathrm{e}, i}$ is the echo amplitude, which 
can be computed using the radar range equation [1] and the constant $c$ in (5) and (6) is the speed of light.

The receiver sees a disturbed version of the echo signal (4). In the following, we assume real zero mean additive white gaussian noise (AWGN) for the disturbance modeled by the stochastic process $n(t)$ :

$$
r_{i}(t)=e_{i}(t)+n(t)
$$

\section{HOMODYNE RECEIVER AND NON-COHERENT DETECTOR}

In this section, the conventional processing of continuous wave radar signals at the receiver, which consists of stretch processing [4] and amplitude detection [1], is briefly summarized. We are assuming a digital baseband processor.

\section{A. Stretch Processing: Downconversion to Complex Baseband}

The LFM waveform can be downcoverted to complex baseband using a homodyne receiver architecture, where the received high frequency signal is mixed directly with the transmitted signal and with a $90^{\circ}$ phase shifted version thereof, a technique known as stretch processing [4]. This leads to the complex baseband signal

$$
\begin{aligned}
q_{i}(t) & =\operatorname{LPF}_{f_{\mathrm{b}, \max }}\left\{\left(s_{i}(t)+j s_{i}\left(t-\tau_{\frac{\pi}{2}}\right)\right) r_{i}(t)\right\} \\
& =\frac{a_{\mathrm{s}, i} a_{\mathrm{e}, i}}{\sqrt{2}} \mathrm{e}^{-j 2 \pi\left(\left(f_{\mathrm{D}, i}+\alpha \tau\right) t+\left(\frac{\alpha \tau}{2}+f_{\mathrm{D}, i}-f_{i}\right) \tau\right)}+z_{i}(t) \\
& \approx a_{\mathrm{y}, i} \mathrm{e}^{-j 2 \pi\left(\left(f_{\mathrm{D}, i}+\alpha \tau\right) t+\left(f_{\mathrm{D}, i}-f_{0}\right) \tau\right)}+z_{i}(t) \\
& \triangleq y_{i}(t)+z_{i}(t)
\end{aligned}
$$

In (8), $\operatorname{LPF}_{f_{\mathrm{b}, \max }}\{\cdot\}$ denotes the filtering with a low pass filter (LPF), which has a passband reaching till the maximum expected beat frequency $f_{\mathrm{b}, \max }$ and $j$ denotes the imaginary unit. The term $z_{i}(t)$ in (9) till (11) denotes the complex baseband noise, which is assumed to be a low pass filtered complex zero mean circularly symmetric white gaussian noise process with variance $\sigma^{2}$. Furthermore, the $z_{i}(t)$ are independent and identically distributed, since they originate from different parts of the spectrum. In (10), the residual video phase $-\alpha \tau^{2}$ [4] has been neglected, which is feasible in typical short range scenarios and when the ramp steepness $\alpha$ is moderate.

\section{B. Detection and Parameter Estimation}

Assuming, the detector operates in the digital domain on the complex baseband signal sampled at a rate of

$$
f_{\mathrm{S}}=\frac{1}{T_{\mathrm{S}}} \equiv f_{\mathrm{b}, \max }
$$

Then the sampled signal is

$$
\begin{aligned}
x_{i}(n) & \triangleq y_{i}\left(n T_{\mathrm{S}}\right)+z_{i}\left(n T_{\mathrm{S}}\right) \\
& =a_{\mathrm{y}, i} \mathrm{e}^{-j 2 \pi\left(\left(f_{\mathrm{D}, i}+\alpha \tau\right) n T_{\mathrm{S}}+\left(f_{\mathrm{D}, i}-f_{i}\right) \tau\right)}+z_{i}\left(n T_{\mathrm{S}}\right) .
\end{aligned}
$$

If the sampling frequency is chosen according to (12), then the complex noise samples $z_{i}\left(n T_{\mathrm{S}}\right)$ are uncorrelated from each other [1].

The parameters of interest in (14) are amplitude $a_{\mathrm{y}, i}$, beat frequency $f_{\mathrm{b}, i} \triangleq f_{\mathrm{D}, i}+\alpha \tau$ and phase $\phi_{\mathrm{y}, i} \triangleq\left(f_{\mathrm{D}, i}-f_{i}\right) \tau$ of the complex sinusoid, all which can be estimated using spectral estimation techniques [5]. For example by calculating the complex spectrum using a normalized N-point FFT:

$$
X_{i}(k)=\frac{1}{N} \mathrm{FFT}_{N}\left\{x_{i}(n)\right\}
$$

In the case of a single target,

$$
\hat{k}=\arg \max _{k}\left|X_{i}(k)\right|,
$$

yields the maximum likeliehood (ML) estimate of the target beat frequency when phase is unknown [6]. In multitarget scenarios, threshold detection performed by comparing the amplitude of the complex spectrum with a threshold $\tilde{\gamma}$ yields nearML performance, when the targets are sufficiently separated [6]. This is called the linear detector [1]. Denoting the hypothesis that a target is present by $H_{1}$ and the Nullhypothesis, which declares that no target thus only noise is present, by $H_{0}$, then the detection rule of the linear detector can be written as

$$
\left|X_{i}(k)\right| \underset{H_{1}}{\stackrel{H_{0}}{\lessgtr}} \tilde{\gamma} .
$$

Another suboptimal detector is the square law detector [1], which decides based on the power estimate of the signal samples

$$
\left|X_{i}(k)\right|^{2} \underset{H_{1}}{\stackrel{H_{0}}{\lessgtr}} \gamma
$$

with the threshold $\gamma \neq \tilde{\gamma}$.

The other two parameters of interest in (14) can be estimated from the frequency estimate [6]:

$$
\begin{aligned}
& \hat{a}_{\mathrm{y}}=\left|X_{i}(\hat{k})\right| \\
& \hat{\phi}_{\mathrm{y}}=\angle X_{i}(\hat{k})
\end{aligned}
$$

\section{Receiver Operating Characteristic}

The performance of a detector is determined by its probability of detection $P_{\mathrm{D}}$ and its probability of false alarm $P_{\mathrm{F}}$ at a given $\mathrm{SNR}$, where

$$
\begin{aligned}
& P_{\mathrm{D}}=P\left\{E_{1} \mid H_{1}\right\}, \\
& P_{\mathrm{F}}=P\left\{E_{1} \mid H_{0}\right\},
\end{aligned}
$$

with $E_{1}$ being the event that the detector decides for a target being present.

The fundamental dilemma in detector design (how to choose the threshold) is that the two probabilities (21) and (22) are coupled. The receiver operating characteristic (ROC) is a figure of merit for detectors showing the tradeoff between these two probabilities. One common representation is

$$
P_{\mathrm{D}}=f\left(P_{\mathrm{F}}, \mathrm{SNR}, \ldots\right)
$$

where other parameters besides the signal to noise ratio (SNR) are detector specific, such as e.g. integration rules. 


\section{PRoposed Method}

By using (17) or (18) for detection, one discards any information contained in the phase $\angle X_{i}(k)$. Thus, both decision rules are suboptimal.

The dilemma is, that although the phase of the target echo in (15) is deterministic, as its value is not known prior to detection, it is not useful for the detector. In the following, a modification of wafevorm (1) is proposed in order to solve this dilemma and thus improve the ROC while maintaining the same target illumination energy and time as when using the unmodified signal, which hereafter will be referred to as conventional signal.

\section{A. Waveform Modifications}

The proposed waveform, which allows to exploit phase information for detection, will be referred to as M-fold signal. It is derived from the conventional signal (1) by introducing the following modifications:

1) $\quad M$-fold multiple simultaneous transmissions of the conventional signal

2) Reduction of the transmitted power of each copy of the conventional signal by a factor of $M$

In transmitting $M$ copies of the conventional signal simultaneously, any time constraints for target illumination are still met. The power reduction is necessary in order to meet a power constraint and in order to allow a fair comparison with the conventional signal by ensuring the same target illumination energy.

Multiple transmissions of the same signal, which is the main modification proposed, allow comparing the estimated phase values of the resulting multiple echoes, which in turn can be used as an additional detection criterion. For a target present $\left(H_{1}\right)$, the $M$ phase values are correlated, where the correlation coefficient depends on the SNR. The higher the SNR, the higher the correlation and thus the less the differences in value. For no target present $\left(H_{0}\right)$, the $M$ phase values are independent from each other and thus very likely to be of very different value.

The possibilities for multiple simultaneous transmissions of LFM waveforms are limited. Taking into account that separation in the polarization state does not lead to separated echoes if the polarization scattering matrix of the target has non-zero entries off the diagonal and spread spectrum techniques would alter the waveform itself, separation in the frequency domain is the remaining option, which is what we are using.

The M-fold signal consists of $M$ copies of the conventional signal (1), which are separated in frequency domain and scaled in amplitude:

$$
s_{\Sigma}(t) \triangleq \frac{1}{\sqrt{M}} \sum_{i=1}^{M} s_{i}(t)
$$

with $s_{i}(t)$ as in (1) and

$$
f_{i}=f_{0}+i \Delta f
$$

where $\Delta f$ is the frequency difference in between the signal copies and $f_{0}$ the lower frequency bound of the M-fold signal. With definition (24), the energy of the M-fold signal is

$$
E_{T, \Sigma}=\int_{0}^{T}\left|s_{\Sigma}(t)\right|^{2} \mathrm{~d} t=a_{\mathrm{s}, i}^{2} E_{T, i, 1}=E_{T, i},
$$

which is the same as the energy (3) of the conventional signal.

\section{B. Receiver Design}

Assuming a target with the same properties as in sec. II, the echo of the M-fold signal is a superposition of the echoes of each copy of the conventional signal:

$$
e_{\Sigma}(t)=\frac{1}{\sqrt{M}} \sum_{i=1}^{M} e_{i}(t)
$$

which will be superimposed by noise at the receiver:

$$
r_{\Sigma}(t)=e_{\Sigma}(t)+n(t)
$$

where $n(t)$ is a real AWGN process with the same characteristics as in (7).

The receiver processes signal (28) by quadrature mixing with each transmitted signal copy individually and subsequent filtering with a LPF as in (8), yielding

$$
\begin{aligned}
q_{\Sigma, i}(t) & =\operatorname{LPF}_{f_{\mathrm{b}, \max }}\left\{\frac{\left(s_{i}(t)+j s_{i}\left(t-\tau_{\frac{\pi}{2}}\right)\right) r_{\Sigma}(t)}{\sqrt{M}}\right\} \\
& \approx \frac{1}{M} y_{i}(t)+\frac{1}{\sqrt{M}} z_{i}(t),
\end{aligned}
$$

where the approximation in (29) is due to neglecting the RVP and the noise process $z_{i}(t)$ is a complex AWGN process with the same characteristics as in (10).

The subsequent analog digital conversion and FFTprocessing is analogous to (13) and (15) yielding

$$
X_{\Sigma, i}(k)=\frac{1}{N} \mathrm{FFT}_{N}\left\{x_{\Sigma, i}(n)\right\}
$$

with

$$
x_{\Sigma, i}(n) \triangleq \frac{1}{M} y_{i}\left(n T_{\mathrm{S}}\right)+\frac{1}{\sqrt{M}} z_{i}\left(n T_{\mathrm{S}}\right) .
$$

\section{Detector exploiting Amplitude and Phase Information}

In order to make use of both amplitude and phase information, a naive detection strategy consists of the following three steps:

1) Square law detection using the complex sum of the $M$ received spectra

$$
X_{\Sigma}(k) \triangleq\left|\sum_{i=1}^{M} X_{\Sigma, i}(k)\right|^{2} \underset{E_{\mathrm{A}, 1}}{\stackrel{E_{\mathrm{A}, 0}}{\lessgtr}} \gamma,
$$

where $\gamma$ is the detection threshold and $E_{\mathrm{A}, 0}\left(E_{\mathrm{A}, 1}\right)$ is the decision that no target (a target) is present based on the amplitude information.

2) Exploiting the $M$ phase spectra $\angle X_{\Sigma, i}(k)$ for detection by comparing them with each other leads to the 

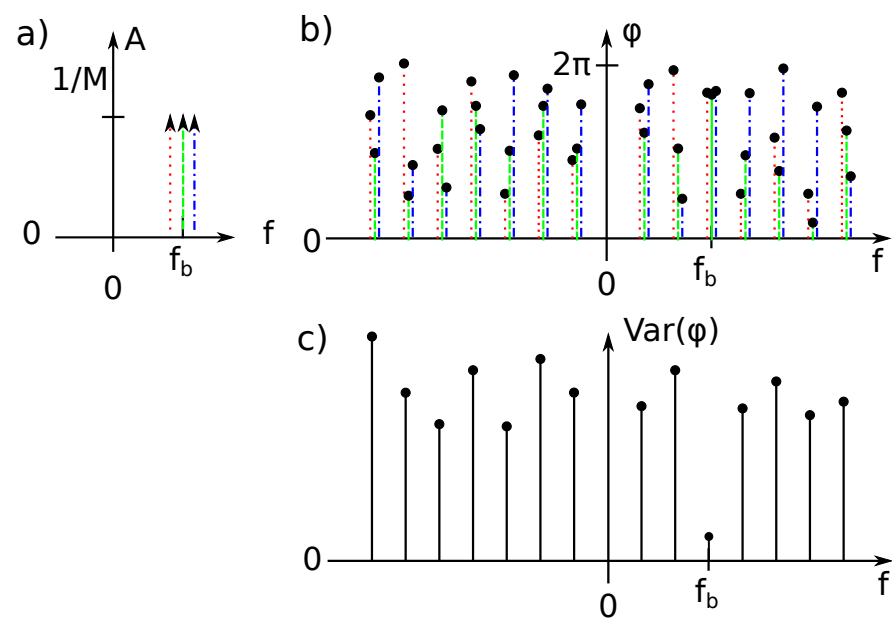

Fig. 1. Effect of calculating the sample variance of the phase values in $M=3$ spectra. In a), three amplitude spectra are shown without noise. The according noisy phases are shown in b) and in c) we can see the effect of calculating the sample variance of the three phase spectra.

decision $E_{\phi, 0}\left(E_{\phi, 1}\right)$ that no target (a target) is present based on the phase information.

3) Combining both decisions to the final detection result.

Under the assumption

$$
M \Delta f \ll f_{i} \Rightarrow f_{\mathrm{D}, 1} \approx f_{\mathrm{D}, 2} \approx \ldots \approx f_{\mathrm{D}, M} \triangleq f_{\mathrm{D}}
$$

and thus

$$
f_{\mathrm{b}, 1} \approx f_{\mathrm{b}, 2} \approx \ldots \approx f_{\mathrm{b}, M} \triangleq f_{\mathrm{b}}
$$

it can be shown that (32) fully recovers the performance of (18), since then, all signal energy is concentrated at $f_{\mathrm{b}}$ and thus the SNR of $X_{\Sigma}(k)$ equals the SNR of $X_{i}(k)$ in (15).

The next question is how to compare the $M$ baseband phase spectra. With the reasoning that the phases of noise only samples are uncorrelated between these spectra, since they originate from different regions of the passband spectrum, but signal plus noise samples are highly correlated between the baseband spectra and thus very likely to have similar values, calculating the sample variance leads to a useful detection statistic. Since the sample mean is not known a priori, we use the unbiased sample variance estimator on the phase samples and define this as estimated phase-variance:

$$
\operatorname{Var}_{\phi, M}(k)=\frac{1}{M-1} \sum_{i=1}^{M}\left(\angle X_{\Sigma, i}(k)-\frac{1}{M} \sum_{i=1}^{M} \angle X_{\Sigma, i}(k)\right)^{2}
$$

The effect of calculating (35) for three $(M=3)$ noisy spectra as defined in (30) is illustrated in fig. 1: the lower $\operatorname{Var}_{\phi, M}(k)$, the higher the probability, that bin $k$ contains a signal sample. Thus, the detection rule using the estimated phase-variance is

$$
\operatorname{Var}_{\phi, M}(k) \underset{E_{\phi, 0}}{\stackrel{E_{\phi, 1}}{\lessgtr}} \gamma_{\phi}
$$

where $\gamma_{\phi}$ is the according threshold.

A combination of (32) and (36) leads to a practical but suboptimal detection rule. Using e.g. logic AND yields

$$
E=E_{\phi} \wedge E_{\mathrm{A}}
$$

a) "AND":

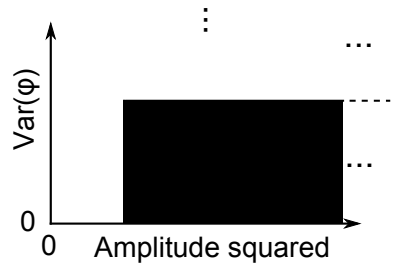

a) "OR":

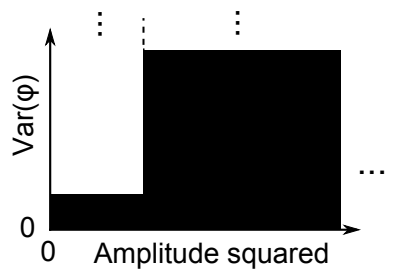

Fig. 2. Suboptimal decisions based on logic AND and logic OR combinations of the two decisions taken based on the amplitude and the phase-variance information lead to rectangular decision regions in the amplitude/phasevariance space. In a), the region for combination by logic AND (37) and in b), the region for combination by logic OR (38) is illustrated. Inside the black area, the decision for presence of a signal is taken, whereas outside, it is decided for signal absence.

and logic OR yields

$$
E=E_{\phi} \vee E_{\mathrm{A}},
$$

where $E$ denotes the final decision. The decision rules (37) and (38) lead to rectangular regions in the amplitude/phasevariance space, which are illustrated in fig. 2 .

An optimal detection rule based on the two decision variables $\operatorname{Var}_{\Phi, M}(k)$ and $X_{\Sigma}(k)$, can be found by deriving the two-dimensional probability density functions (pdfs) $p_{\vec{\Xi}, \mathrm{n}}(\vec{\xi})$ for the case of noise only and $p_{\vec{\Xi}, \mathrm{S}}(\vec{\xi})$ for the case of signal plus noise, where $\vec{\xi}=\left(X_{\Sigma} \operatorname{Var}_{\Phi, M}\right)^{\mathrm{T}}$ with $(\cdot)^{\mathrm{T}}$ denoting the vector transpose. The index $k$ has been dropped intentionally, as it is not needed here. Deriving these probability densities is quite challanging due to the correlation between the two random variables and the fact that the sample variance depends on the sample mean, which is why for a first evaluation of the proposed method, we have resorted to simulations.

\section{Simulations And Analysis}

The evaluation of the proposed method is based on simulations of the baseband signal samples in frequency domain assuming (33) holds. Then (30) can be modeled by a complex circular symmetric gaussian random variable with zero mean in the case of noise only and with a mean equal to the amplitude of the signal including FFT processing gain in the case of signal plus noise. All simulations are based on $10^{7}$ samples and the number of signal copies for the M-fold signal is $M=5$.

A scatter plot of 600 realizations per case (noise only, signal plus noise) of the decision vector $\vec{\xi}$ for a post processing SNR of $6 \mathrm{~dB}$ is shown in fig. 3. It hints, that the proposed method should outperform detectors based on pure amplitude information, if decision regions are well chosen.

This hypothesis is strengthened, when taking a look at the empirical probabiliy density functions for the decision vector at the same SNR of $6 \mathrm{~dB}$, which are depicted in fig. 4 for the case of noise only and for the case of signal plus noise.

Optimal decision regions, which have been found based on the estimated likelihood ratio

$$
\hat{l}(\vec{\xi})=\frac{\hat{p}_{\vec{\Xi}, \mathrm{n}}(\vec{\xi})}{\hat{p}_{\vec{\Xi}, \mathrm{s}}(\vec{\xi})},
$$




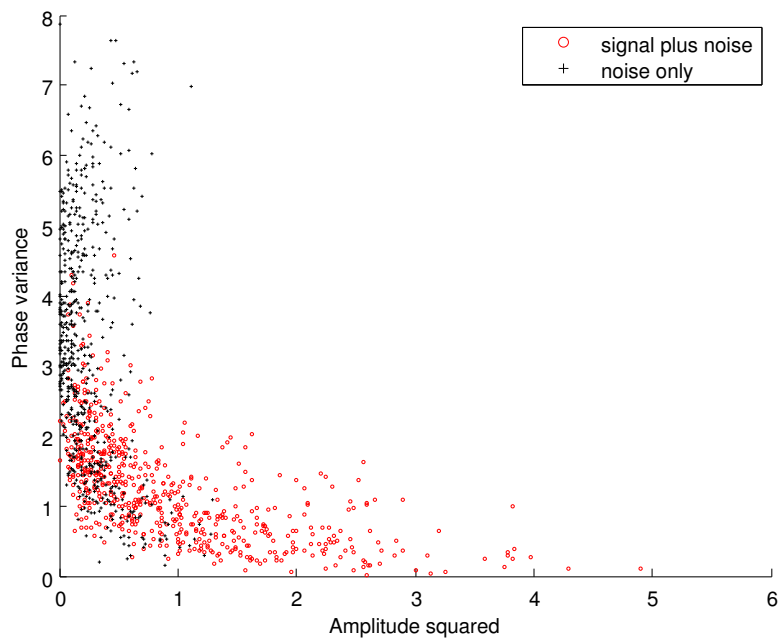

Fig. 3. Scatter plot of 600 realizations per case (noise only, signal plus noise) of the decision vector $\vec{\xi}$ at a SNR of $6 \mathrm{~dB}$ and $M=5$. Black crosses denote noise only samples and red circles signal plus noise samples. It can clearly be seen, that by considering both dimensions, detection performance can be improved compared to considering only amplitude.
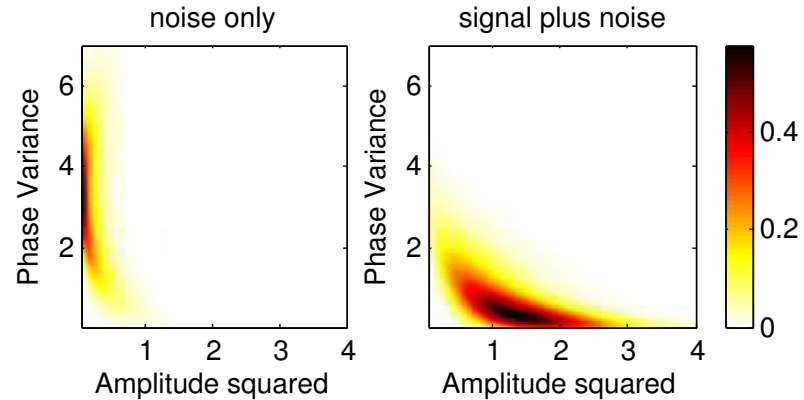

Fig. 4. Empirical probability density functions $\hat{p}_{\vec{\Xi}, \mathrm{n}}(\vec{\xi})$ and $\hat{p}_{\vec{\Xi}, \mathrm{s}}(\vec{\xi})$ of the decision vector $\vec{\xi}$ for both cases (nois only, signal plus noise) at a SNR of $6 \mathrm{~dB}$ and $M=5$.

are shown in fig. 5 for a SNR of $6 \mathrm{~dB}$ and false alarm rates of $10^{-3}, 10^{-2}$ and $10^{-1}$. It can be seen that, as predicted, the regions are not rectangular. Thus, the optimal detection rule for the proposed method is based on areas in the two-dimensional decision space, which cannot be trivially decomposed into two one-dimensional regions.

In order to compare the detectors, ROCs have been derived for various SNRs using the following detectors:

- Square law detector on the conventional signal (18), which will serve as reference

- $\quad$ Square law detector on the M-fold signal (32)

- Detector using only phase information of the M-fold signal (36)

- $\quad$ Detector combining the decisions based on phase (36) and amplitude information (32) of the M-fold signal by logic AND (37)

- $\quad$ Detector combining the decisions based on phase (36) and amplitude information (32) of the $M$-fold signal

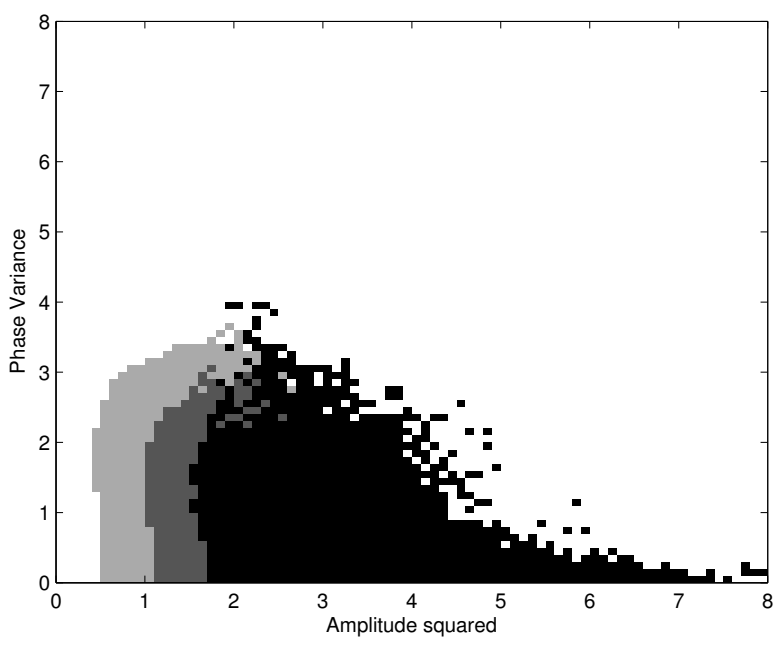

Fig. 5. Optimal decision regions for different false alarm rates at a SNR of $6 \mathrm{~dB}$ and $M=5$, which have been found using likelihood ratios of the empirical probability density functions. The black region yields a false alarm rate (FAR) of $10^{-3}$; if the dark grey region is added, a FAR of $10^{-2}$ is achieved and if furthermore the light grey region is added, a FAR of $10^{-1}$ is achieved.

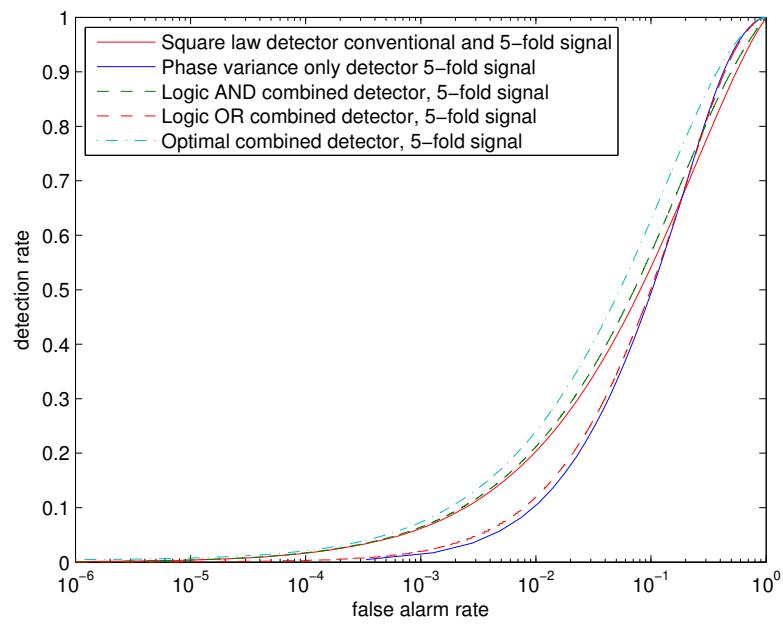

Fig. 6. Receiver Operating Characteristics (ROCs) of 6 different detectors at a SNR of $3 \mathrm{~dB}$. The curve of the square law detector operating on the conventional signal, which serves as reference, resides directly on the curve of the square law detector operating on the 5-fold signal. It can be seen, that the optimal detector for the M-fold signal clearly outperforms the square law detector, achieving nearly $10 \%$ higher detection rates. The sub-optimal logic AND combined detector achieves nearly 3\% gain in detection rate compared to the reference, whereas the logic OR combined detector and the detector based only on phase-variance perform only better than the reference at high false alarm rates.

by logic OR (38)

- Optimal detector for the M-fold signal based on the empirically found decision regions illustrated in fig. 5 .

In fig. 6, it can be seen that at a SNR of $3 \mathrm{~dB}$, the optimal detector for the M-fold signal clearly outperforms the conventional square law detector, achieving nearly $10 \%$ higher DRs. Also, the sub-optimal logic AND combined detector achieves better performance (about 3\% gain in DR) than 


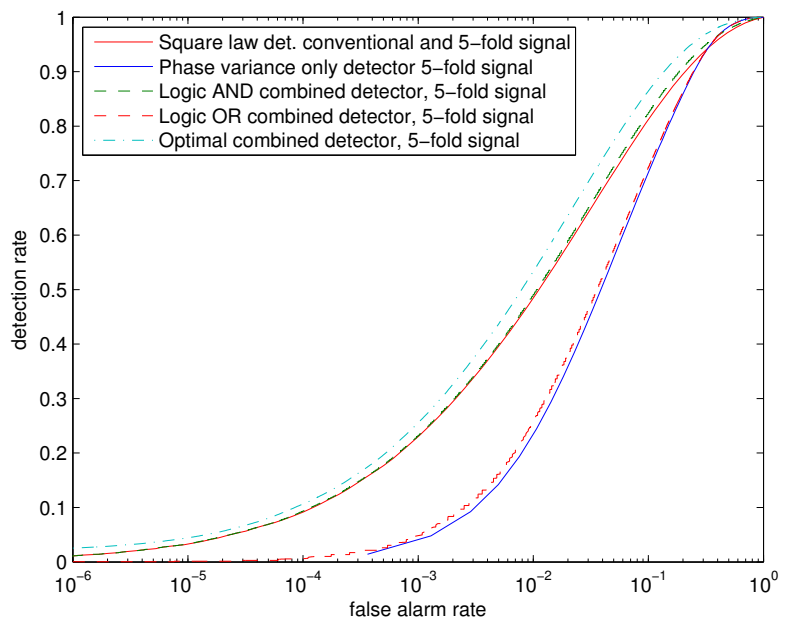

Fig. 7. ROCs of 6 different detectors at a SNR of $6 \mathrm{~dB}$. The curve of the square law detector operating on the conventional signal, which serves as reference, resides directly on the curve of the square law detector operating on the 5-fold signal. The performance gain of the optimal combined detector for the M-fold signal reaches about 5\% compared to the reference, whereas the logic AND combined detector performs just slightly better than the reference and the other suboptimal detectors perform clearly inferior except at very high false alarm rates.

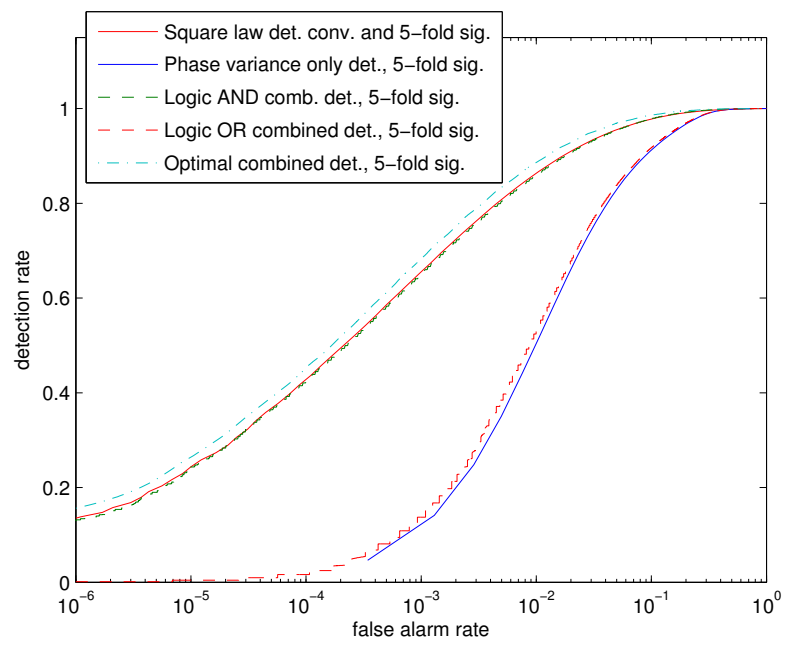

Fig. 8. ROCs of 6 different detectors at a SNR of $9 \mathrm{~dB}$. The curve of the square law detector operating on the conventional signal, which serves as reference, resides directly on the curve of the square law detector operating on the 5-fold signal. The optimal detector for the M-fold signal achieves a gain of $2-3 \%$ in detection rate compared to the reference. The logic AND combined detector performs slightly worse than the reference. The other suboptimal detectors perform very poorly as compared to the reference.

the conventional square law detector, whereas the logic OR combined detector and the detector based only on phasevariance show performance improvements only at high false alarm rates (FARs). These findings are generally confirmed by fig. 7, which shows ROCs of the same detectors at a SNR of $6 \mathrm{~dB}$. However, the performance gain of the optimal combined detector for the M-fold signal is reduced compared to its performance at $3 \mathrm{~dB}$ SNR to about 5\%. The logic AND combined detector performs just slightly better than the reference. At an even higher SNR of $9 \mathrm{~dB}$, at which ROCs are shown in fig. 8, the performance gain of the optimal combined detector drops further to about $2-3 \%$ compared to the conventional square law detector, whereas the logic AND combined detector performs slightly worse than the reference and the other suboptimal detectors fall clearly off except at very high FARs $\left(>10^{-1}\right)$. The reduction of performance gain with rising SNR can be explained by the rising correlation of the two decision variables amplitude and phase-variance in the case of a signal being present.

Summarized, the optimal detector for the M-fold signal achieves a gain in DR compared to the conventional square law detector of nearly $10 \%$ at a SNR of $3 \mathrm{~dB}$. This performance gain drops to about $5 \%$ at a SNR of $6 \mathrm{~dB}$ and it drops even further to about $2-3 \%$ at a SNR of $9 \mathrm{~dB}$. This behaiviour can be explained by the two pdfs $p_{\vec{\Xi}, \mathrm{n}}(\vec{\xi})$ and $p_{\vec{\Xi}, \mathrm{s}}(\vec{\xi})$. They become more and more separated in amplitude as the SNR rises. Thus, at higher SNR less information can be gained by including the phase variance in the decision process.

Furthermore, the logic AND combined detector achieves smaller gains in DR than the optimal combined detector but shows the same behavior as SNR rises. The performance gain of the logic AND combined detector vanishes at a SNR of $9 \mathrm{~dB}$. The other suboptimal detectors perform clearly inferior at all observed SNR values except at very high FARs $\left(>10^{-1}\right)$, thus should only be considered when this is acceptable.

\section{CONCLUSION}

The proposed signal modifications allow exploiting phase information in a (frequency modulated) continuous wave radar at the detector, while ensuring the same target illumination energy and time as when using a conventional waveform. It has been shown how this additional information can be used to enhance detector performance. The performance gain was evaluated by simulation for an optimal and three suboptimal detectors. The optimal detector and one suboptimal detector outperform the conventional square law detector especially in the low SNR regime, where a gain of about $10 \%$ in detection rate is achieved by the optimal detector. The performance gain diminishes as SNR rises, which is due to the increasing amplitude separation of the two probability densities.

The results are of interest, as it is shown for the first time, how phase information can be exploited for detection in any kind of continuous wave radar and that doing so leads to a performance gain especially at low signal to noise ratios, where ROC improvements are particularly valuable.

\section{REFERENCES}

[1] M. A. Richards, Fundamentals of Radar Signal Processing, 2nd ed. New York: McGraw-Hill, 2014.

[2] B. Atayants, V. Davydochkin, V. Ezerskiy, V. Parshin, and S. Smolskiy, Precision FMCW Short-Range Radar for Industrial Applications. Norwood, MA: Artech House, 2014.

[3] A. Stove, "Linear fmcw radar techniques," Radar and Signal Processing, IEE Proceedings F, vol. 139, no. 5, pp. 343-350, Oct 1992.

[4] W. L. Melvin and J. A. Scheer, Principles of Modern Radar. Edison, NJ: Scitech Publishing, 2013, vol. Vol. II: Advanced Techniques.

[5] P. Stoica and R. Moses, Spectral Analysis of Signals. Upper Saddle River, NJ: Prentice-Hall, 2005.

[6] S. M. Kay, Modern spectral estimation: Theory \& application. Englewood Cliffs, NJ: Prentice Hall Signal Processing Series, 1988. 